\title{
Les troubles du cycle de la sportive. Diagnostic et prise en charge
}

\author{
Carole Maître
}

Service médical de l'INSEP, 11, avenue du Tremblay, 75012 Paris, France

A paraître dans Science \& Sports, 2013

\section{Résumé}

Les troubles du cycle de la sportive sont induits par une disponibilité énergétique insuffisante pour le fonctionnement de l'axe hypothalamo-hypophysaire (AHH). De nombreux médiateurs endocriniens et des neurotransmetteurs en particulier la leptine, le peptide YY (PYY), la ghreline et le système kisspeptine interviennent pour ralentir directement ou non la pulsatilité de $\mathrm{GnRH}$, et la production des stéroïdes ovariens. Cet état d'hypométabolisme vise à orienter la dépense énergétique vers les organes vitaux et les muscles en fonctionnement avec en corollaire une perturbation du métabolisme osseux, un risque d'ostéoporose ou d'ostéopénie et une altération de la fonction endothéliale avec un risque majorée d'athérosclérose. Il doit être pris en charge précocement pour préserver la santé de la sportive.

Mots clés: Troubles du cycle; Aménorrhée; Triade de l'athlète; Densité osseuse; Trouble du comportement alimentaire; Fonction endothéliale

\section{Summary}

Menstrual disturbances are induced by insufficient energy availability for functioning of pituitary axis. Many endocrine mediators and neurotransmitters as leptin, peptide YY (PYY), ghrelin and kisspeptin system involved directly or indirectly to slow pulsatile $\mathrm{GnRH}$, and production of ovarian steroids. This state of hypometabolism is intended to guide the energy expenditure to vital organs and muscles functioning in corollary with a disturbance of bone metabolism, risk of osteoporosis or osteopenia and impaired endothelial function with increased risk of atherosclerosis. It should be prisen load early to preserve the health of the athletes.

Keywords: Menstrual disturbances; Amenorrhea; Female athlete triad; Bone density; Eating disorder; Endothelial function 
Depuis les années 1990, la connaissance de la physiopathologie des troubles du cycle observés chez la sportive a permis de substituer à la notion de triade de l'athlète constituée par l'aménorrhée, les troubles du comportement alimentaire et l'ostéoporose, un modèle de dysfonctionnement fait d'un continuum de perturbations du cycle, de disponibilité énergétique en défaut et de densité osseuse diminuée [1] and [2]. Le générateur hypothalamique, par la pulsatilité ordonnée des sécrétions de GnRH, est garant des sécrétions rythmiques de LH et FSH hypophysaire, assurant maturation folliculaire, cycles et ovulation mais, il est soumis à l'impact des rétrocontrôles de régulation, à l'homéostasie des fonctions neuroendocrines adrénergique et thyroïdienne, des endocannabinoïdes, des cytokines et dépend tout particulièrement chez la sportive du niveau de disponibilité énergétique, une balance énergétique négative impacte la fonction gonadotrope et le métabolisme osseux.

\section{Les troubles du cycle de la sportive}

Les troubles du cycle sont la traduction clinique du ralentissement de l'axe hypothalamo-hypophysaire (AHH) avec de façon chronologique apparition d'une baisse de la sécrétion ovarienne de progestérone qui caractérise l'insuffisance lutéale et secondairement d'une insuffisance estrogénique.

\subsection{Définitions et description}

L'insuffisance lutéale ou insuffisance progestative de la deuxième partie du cycle, elle est responsable de cycles courts de moins de 24 jours. Elle est asymptomatique ou s'accompagne de syndrome prémenstruel qui peut être marqué par un ou plusieurs symptômes physiques liés directement à l'hyperestrogénie relative tels la rétention hydrique, la prise de poids, les mastodynies, l'hyperlaxité ligamentaire, les crampes, les céphalées, la fatigue, et des symptômes psychiques tels l'irritabilité, les troubles du sommeil et la sensibilité accrue au stress et enfin une tendance dépressive. Ces symptômes apparaissent gêner la performance et entraîner une absence aux séances d'entraînement, d'autant que peut s'y associer une dysménorrhée d'intensité variable [3] and [4]. C'est un état favorisant les régimes alimentaires inappropriés lors de la période prémenstruelle essentiellement dans les sports à catégories de poids où la pesée est plus fréquente :

- l'oligoménorrhée, la spanioménorrhée est l'allongement des cycles de plus de 35 jours ;

- l'aménorrhée : il peut s'agir d'aménorrhée primaire qui correspond à une absence de ménarche après l'âge de 15 ans, alors même que les caractères sexuels sont présents, soit une aménorrhée secondaire, absence de règles de trois mois ou plus, apparaissant après la ménarche. Il s'agit d'une aménorrhée hypothalamique fonctionnelle, liée au ralentissement de l'AHH avec pulsatilité diminuée de la LH, sécrétion plus faible de FSH, ralentissement du cycle ovarien de la folliculogénèse. Elle est rarement un motif de consultation chez la sportive, car elle est bien acceptée du fait de l'absence de symptômes, ce qui est à l'origine de retard à la prise en charge et d'effets délétères sur le métabolisme osseux et lipidique avec des conséquences sur la solidité osseuse et la fonction endothéliale.

L'anovulation peut être présente à tous les stades de cette cascade de dysfonctionnements.

\subsection{La prévalence des troubles du cycle}

Les troubles du cycle varient en fonction du type de sport pratiqué et du niveau de pratique susceptible d'engendrer un déséquilibre de la balance énergétique en l'absence d'adaptation nutritionnelle suffisante. Leur prévalence atteint 4 à $79 \%$ chez les sportives [5] ; les sports les plus concernés sont :

- les sports à catégories de poids, judo, karaté, boxe, lutte, haltérophilie, aviron ; 
- les sports dits esthétiques où la minceur est recherchée, natation synchrone, mais aussi patinage, gymnastique rythmique et artistique, sports à début prépubertaire qui peuvent s'accompagner de retard de la ménarche jusqu'à deux ans par rapport à une population sédentaire avec une prévalence des troubles du cycle de 30 à $79 \%$ [6] ;

- les sports d'endurance, athlétisme, course à pied, cyclisme sur route où le poids peut être vécu comme une limite à la performance avec une prévalence de 26 à $35 \%$.

L'insuffisance lutéale et l'anovulation se retrouve chez près de la moitié des sportives, tout sport confondu, l'aménorrhée est présente chez $7 \%$ des sportives dans une population de 400 sportives françaises de haut niveau ayant répondu à un questionnaire sur les troubles du cycle, à durée d'entraînement égal, mais peut atteindre $30 \%$ des sportives [3]. Dans la population générale, l'aménorrhée varie de 2 à $5 \%$.

La prévalence des troubles du cycle n'augmente pas avec le volume d'entraînement : sur une population de 669 sportives norvégiennes de haut niveau, il n'est pas noté de différence significative entre le volume d'entraînement le plus élevé et le plus faible et il n'y a pas plus de perturbations chez les sportives des sports techniques ou collectifs suivant un entraînement moyen de 14 heures par semaine que dans la population sédentaire [7]. Ce n'est ni l'intensité, ni le volume d'entraînement qui est à l'origine du ralentissement de l'AHH, mais une réserve énergétique faible : les sports avec la prévalence la plus élevée ont en dénominateur commun une masse grasse faible, reflet d'une réserve énergétique insuffisante pour assurer le fonctionnement de l'AHH. La masse grasse est mesurée aux six plis cutanés ou mieux par absorptiométrie biphotonique (DXA). L'indice de masse corporelle (IMC) n'est pas un bon indicateur dans une population sportive, car elle ne reflète pas la composition corporelle.

\section{La disponibilité énergétique}

La disponibilité énergétique par 24 heures est la différence entre l'apport énergétique nutritionnel assimilé sur 24 heures et la dépense énergétique liée à la pratique sportive sur 24 heures : une disponibilité énergétique basse induit une réponse d'hypométabolisme, réponse biologique d'épargne énergétique pour assurer le maintien des fonctions vitales [2]. Ce déséquilibre peut être rapporté à un déficit calorique quantitatif global ou qualitatif en lipides dont la ration journalière peut chez les sportives être inférieure à $15 \%$.

Ce déficit énergétique peut être modéré, temporaire ou chronique, entrant dans la nosologie des troubles du comportement alimentaire dont la prévalence dans la population sportive élite est de 25 à $31 \%$ alors que dans la population générale la prévalence de ces troubles est de 5,5 à $9 \%$ [8]. Des facteurs de vulnérabilité peuvent être recherchés, dans les sports à catégories de poids : pesée fréquente, période précompétitive ou difficulté à rester dans sa catégorie de poids, surentrainement, difficulté à gérer les émotions (stress de la compétition, blessures, défaite, relations avec l'entourage, médiatisation excessive), prédisposition individuelle (perfectionnisme) associée aux contraintes de disciplines en début prépubertaire. La perte de poids dans un but d'améliorer la performance s'accompagne de restriction calorique, et peut initier de troubles du comportement alimentaire et un déficit énergétique chronique. Mais un déficit énergétique peut être involontaire lié à une dépense énergétique non compensée par l'alimentation ou du fait d'un régime végétarien strict par défaut d'éducation nutritionnelle. Depuis les travaux de Hilton et Loucks, il est établi que le déficit énergétique est responsable du ralentissement de la fonction gonadotrope, affectant le générateur 
hypothalamique et la pulsatilité de la LH avec une fréquence de pulse plus grande et une amplitude plus faible [9]. Un seuil minimal de disponibilité énergétique de $30 \mathrm{kcal}$ par jour et par $\mathrm{kg}$ de masse maigre est nécessaire au fonctionnement de l'ensemble des fonctions y compris la fonction de la reproduction [10].

\section{Les modifications hormonales sont les conséquences d'une faible disponibilité énergétique}

La leptine est l'hormone clef qui assure le relais entre le déficit énergétique, la masse grasse et la fonction gonadotrope, peptide de 167 acides aminés secrétée directement par le tissu adipeux, mais aussi par l'hypothalamus, l'hypophyse, le placenta, le foie, l'estomac et le muscle. Sa transcription est stimulée par l'insuline, elle diminue avec le volume des adipocytes : une quantité moindre de triglycérides pourrait induire une diminution de l'expression du gène $\mathrm{Ob}$ dans la cellule adipeuse [11].

Hormone - signal de la satiété, elle a de nombreux récepteurs et intervient sur les fonctions neuroendocrines importantes, modulant l'activité des axes thyréotrope, somatotrope, corticotrope et gonadotrope (récepteurs sur l'hypothalamus et l'ovaire), module la formation osseuse par ses récepteurs sur les cellules préostéoblastiques [12] and [13]. Son taux plasmatique s'élève progressivement durant la phase prépubertaire et est un élément initiateur de la puberté [6].

Hilton et Loucks ont montré que chez la femme sédentaire la leptinémie est plus basse dans le groupe carencé en apports alimentaires par rapport au groupe de femmes non carencées, plus bas chez les athlètes de haut niveau en déficit énergétique que chez les sédentaires, même si les cycles sont encore présents : les modifications hormonales, traduisant un état d'hypométabolisme précèdent l'expression clinique ; en cas d'aménorrhée, une abolition du cycle nycthéméral de la leptine est observée [9]. L'administration exogène de leptine ne rétablit les cycles menstruels qu'en présence d'une disponibilité en substrats énergétiques, en glucose par exemple, suffisante. La leptine joue un rôle essentiel à la fonction de la reproduction sous le contrôle du couple insuline-glucose.

Les autres marqueurs du déficit énergétique contribuent au ralentissement du cycle avec pour la plupart un parallélisme entre les perturbations biologiques, la disponibilité énergétique et les perturbations du cycle :

- l'IGF1 abaissé agit en synergie avec la baisse de leptine ralentissant le cycle. In vitro, l'IGF1 stimule la sécrétion pulsatile de GnRH et stimule le relargage de la LH hypophysaire ;

- le neuropeptide Y (NPY) produit dans le noyau arqué de l'hypothalamus voit son action freinée par la balance énergétique négative or le NPY stimule la sécrétion pulsatile de GnRH hypothalamique [14];

- le peptide YY (PYY) produit par les cellules gastro-intestinales appartient à la même famille que le précédent, son taux corrélé au déficit énergétique, est significativement plus élevé chez les athlètes en aménorrhée par rapport à celles en euménorrhée, il agit en inhibant la sécrétion de NPY au niveau hypothalamique (récepteurs Y2 sur le noyau arqué) et contribue ainsi au ralentissement de l'axe gonadotrope, son taux est un facteur prédictif de l'hypotestostéronémie, les modèles animaux ont montré une stimulation par la testostérone du relargage de GNRH via son action sur les neurones GABA, ce qui suggère un effet freinateur complémentaire de l'hypotestostéronémie sur l'AHH chez la sportive en aménorrhée. Le PYY a de plus un rôle direct sur le métabolisme osseux par ses récepteurs Y2 sur les ostéoblastes, un taux élevé étant inversement corrélé aux marqueurs de l'ostéoformation (procollagène) et 
cela agissant en synergie avec la diminution d'IGF1, de leptine et d'estrogène sur le turnover osseux, aux dépens de la densité minérale osseuse (DMO) [13] and [14];

- la ghreline (produite par les cellules fundiques de l'estomac et le noyau arqué hypothalamique), hormone peptidique orexigène, a une concentration significativement plus élevée chez les sportives en aménorrhée par rapport à celles en euménorrhée, elle inhibe la sécrétion de $\mathrm{GnH}$ et est inversement corrélée à la masse grasse [12] and [13] ;

- ces médiateurs endocriniens dont l'expression est modifiée par la balance énergétique négative ont une action soutenue par le neuromédiateur kisspeptine présent dans l'hypothalamus (noyau arqué et noyau paraventriculaire), sa fixation sur son récepteur GPR54 au niveau des neurones à GnRH stimule la sécrétion pulsatile de GnRH, et joue le rôle d'intégrateur au niveau hypothalamique des boucles de rétrocontrôles positive ou négative suivant le contexte stéroïdien, or l'expression du gène kiss est diminuée par une balance énergétique négative et stimulée par la leptine, effondrée chez la sportive avec une disponibilité énergétique insuffisante [15];

- la tri-iodotyronine (TT3) est diminuée, parallèlement à la diminution de la disponibilité énergétique par kilo de masse maigre ; cette diminution est observée non seulement chez les sportives en aménorrhée mais aussi au cours de perturbations modérées comme l'insuffisance lutéale de la sportive, c'est le métabolisme oxydatif de la cellule musculaire qui est perturbé, limitant les possibilités physiologiques de récupération [5];

- la sécrétion de cortisol est augmentée de façon significative chez les sportives en aménorrhée par rapport aux sportives en euménorrhée, en rapport avec la dépense énergétique, la baisse des substrats énergétiques et le stress par stimulation de l'axe corticotrope, elle est corrélée inversement au taux de LH, et cela de façon, en partie, indépendante du taux de leptine et de ghreline, limitant la pulsatilité de LH [16].

Ainsi s'établit une relation dose-réponse entre les paramètres biologiques du statut énergétique de la sportive et les différentes catégories de troubles du cycle suggérant qu'une variation même modérée en disponibilité énergétique peut perturber l'AHH, ralentir la maturation folliculaire et compromettre la phase lutéale [10]. Ces ajustements métaboliques visant à épargner le métabolisme énergétique surviennent avant la survenue de l'aménorrhée, ce qui explique également les délais de reprise des cycles réguliers (délai moyen de 11 mois) après prise en charge par une adaptation nutritionnelle à la dépense énergétique.

En pratique, devant une aménorrhée ou une oligoménorrhée de la sportive, le bilan hormonal comprend :

- le dosage des taux plasmatiques de LH, FSH, estradiolémie abaissés confirmant l'origine hypothamo-hypophysaire ;

- un dosage de $17 \mathrm{OH}$ progestérone normal permettant d'exclure un bloc enzymatique en 21 hydroxylase dans une forme non classique d'hyperplasie des surrénales à révélation tardive accompagnée de symptômes cliniques d'hyperandrogénie, les dosages de testostéronémie, SDHEA et de delta 4 androstènedione compléteront le bilan d'une hyperandrogénie clinique en distinguant la dystrophie polykystique ovarienne (ratio LH sur FSH supérieur à 2), d'une tumeur ovarienne (Delta 4 androstènedione) ou surrénalienne (SDHEA). 
Le dosage de la TSH recherchant un dysfonctionnement primaire de l'axe thyréotrope et le dosage de la prolactine un prolactinome hypophysaire.

Un dosage des bêta-HCG plasmatiques indispensable devant un trouble du cycle récent et a fortiori devant une aménorrhée pour éliminer un début de grossesse.

Le diagnostic d'aménorrhée fonctionnelle est obligatoirement un diagnostic d'élimination [17].

\section{Les conséquences des troubles du cycle}

\subsection{De l'ostéopénie à l'ostéoporose}

L'hypoestrogénie intervient en augmentant le turnover physiologique formation-réduction osseuse aux dépens de l'ostéogenèse, le déficit énergétique via la baisse de l'IGF1, la T3 et l'insuline intervient également sur les marqueurs de l'ostéoformation : l'ostéocalcine puis le peptide procollagène de type I sont diminués avec un effet dose réponse fonction de la disponibilité énergétique, la résorption osseuse augmente seulement pour une disponibilité énergétique très basse, inférieure à $10 \mathrm{kcal} / \mathrm{kg}$ de masse maigre par jour[18]. Les autres marqueurs de la balance énergétique négative comme les taux de cortisol, leptine, PYY ont également un rôle délétère sur la densité osseuse [19] and [20].

Les cycles irréguliers sont associés chez la sportive à une diminution de la densité osseuse sur les sites osseux non portés ou portés à forte densité trabéculaire, avec un risque aggravé de fractures de fatigue (incidence multipliée par 2 à 4), risque d'autant plus grand que l'hypoestrogénie est prolongée dans le temps, la présence de récepteur alpha estrogéniques sur les mécano transducteurs peut expliquer l'incapacité progressive des ostéocytes à répondre aux stimulations de l'impact au sol des sports portés avec accumulation de microtraumatismes et fracture de fatigue dont la survenue est tributaire aussi de la micro-architecture osseuse et la qualité osseuse. Les conséquences à long terme sur la densité osseuse sont en partie irréversibles d'autant que les troubles ont été précoces et justifie les préconisations de l'American College of Sports Medicine concernant la mesure de la DMO par la DEXA proposée devant toute aménorrhée de plus de six mois, et ou une ou plusieurs fractures de fatigue et, ou un trouble des conduites alimentaires avec une réévaluation à 1 an comparant l'évolution de la DMO sur le Z-score établi par le même appareil, devant la persistance d'un symptôme ou en cas de DMO basse (Z-score inférieure à 1) [21] and [22]. Le risque de fracture de fatigue est expliqué à la sportive pour améliorer l'adhésion à une prise en charge nutritionnelle.

\subsection{L'infertilité}

L'estradiolémie effondrée et l'absence de maturation folliculaire s'accompagne d'une anovulation. Une étude récente concernant 3887 femmes norvégiennes de moins de 45 ans rapporte qu'un indice d'activité physique élevé (intensité, durée, fréquence) après ajustement des variables (âge, parité, tabagisme et statut marital) tend à majorer le risque d'infertilité par rapport aux femmes ayant un indice d'activité modérée ou légère (OR : 1,5 ; IC 95 : 1-2,3). Dans le sous -groupe des femmes de moins de 30 ans, la relation activité physique et infertilité suit une courbe en $U$ avec une augmentation de la pathologie aux pôles extrêmes de la pratique $(<15$ min par jour et $>60$ minutes par jour) [23].

\subsection{La perturbation de la fonction endothéliale}

Sous l'effet du déficit en estrogène, les effets protecteurs des estrogènes sur la fonction endothéliale sont perturbés avec des effets à court terme comme la diminution de la production endothéliale de monoxyde d'azote (NO), la diminution de la vasodilatation chez les sportives en aménorrhée, des 
effets à long terme génomiques concernant la transcription et l'activité enzymatique endothéliale NO synthase. La diminution de la vasodilatation à deux effets : la diminution des capacités musculaire et l'augmentation du risque d'athérosclérose chez ces sportives en aménorrhée, d'autant qu'un profil lipidique apparaît modifié avec augmentation du cholestérol total, du LDL cholestérol, associée ou non à une diminution d'HDL [24] and [25]. Ce risque est apparu diminué chez des athlètes en aménorrhée après neuf mois de traitement par estroprogestatifs (EP) dits de seconde génération au levonorgestrel [26].

\section{La prise en charge des troubles du cycle de la sportive. Place de la contraception estroprogestative}

La prise en charge repose sur une équipe médicale (gynécologue, endocrinologue, physiologistemédecin du sport, nutritionniste) en lien avec la sportive et l'entraîneur et vise à restaurer des cycles menstruels en équilibrant la dépense énergétique à l'apport énergétique alimentaire, le but étant de retrouver une disponibilité énergétique de $45 \mathrm{kcal} / \mathrm{kg}$ de masse maigre et par jour ce qui peut être un vrai challenge dans certains sports où la minceur sont un facteur apparent de performance. L'information à la sportive concernant les risques des troubles du cycle tout particulièrement le risque osseux, un suivi psychologique en cas de troubles des conduites alimentaires, un environnement sportif et familial soutenant sont importants.

Un apport protéique suffisant (au moins $1 \mathrm{~g} / \mathrm{kg}$ de poids corporel), un apport calcique (1000-1300 $\mathrm{mg} /$ jour$)$ et en vitamine D (400-800 UI/jour) sont recommandés [2] and [17].

Les bénéfices sur la DMO d'une supplémentation en estrogène par l'intermédiaire d'une prescription de contraception EP sont contradictoires, la prescription d'EP dans un but de compensation hormonale sera réservée aux sportives chez lesquelles la restauration des cycles n'est pas obtenue faute d'un équilibre adéquat entre les apports et la dépense énergétique : un gain faible a pu être observé, voire une stabilisation de la DMO. Ces résultats inconstants peuvent être liés aux multiples modifications neurohormonales liées à la disponibilité énergétique qui agissent sur le métabolisme osseux, indépendamment de l'hypoestrogénie.

La survenue de fracture de fatigue sous contraception EP doit faire évaluer le statut nutritionnel (y compris un dosage en vitamine D) et la balance énergétique.

Dans le choix de contraceptif EP, et selon les recommandations actuelles, les EP de seconde génération seront prescrits en première intention dans le respect des contre-indications et après bilan du métabolisme lipidique.

\section{Conclusion}

Le diagnostic d'oligoménorrhée ou d'aménorrhée liée à une disponibilité énergétique faible, avec ou sans troubles du comportement alimentaire est souvent tardif et implique une prise en charge globale pour préserver la santé de la sportive. L'information de la sportive et de son environnement sportif quant aux risques sur le métabolisme osseux et la fonction endothéliale est essentielle à la prévention, l'éducation nutritionnelle constitue le premier volet de la thérapeutique.

\section{Déclaration d'intérêts}

L'auteur déclare ne pas avoir de conflits d'intérêts en relation avec cet article. 


\section{Références}

[1] A. Nativ, R. Agostini, B. Drinkwater. The female athlete triad: the inter-relatedness of disordered eating, amenorrhea and osteoporosis. Clin Sports Med, 13 (2) (1994), pp. 405-418

[2] A. Nattiv, A.B. Loucks, M.M. Manore et al. The female athlete triad. Med Sci Sports Exerc, 39 (10) (2007), pp. 1867-1882

[3] C. Maître. Troubles du cycle de la sportive de haut niveau. Cah INSEP, 41 (2008), pp. 165-174

[4] A.J. Daley. Exercise and primary dysmenorrhoea: a comprehensive and critical review of the literature. Sports Med, 38 (8) (2008), pp. 659-670

[5] M.J. De Souza, R.J. Toombs, Scheid J.L. et al. High prevalence of subtle and severe menstrual disturbances in exercising women: confirmation using daily hormone measures. Hum Reprod, 25 (2) (2010), pp. 491-503

[6] K. Klentrou, M. Plyley. Onset of puberty, menstrual frequency and body fat in elite rhythmic gymnasts compared with normal controls. Br J Sports Med, 37 (2003), pp. 490-494

[7] Torstveit, Sundgot, Borgen. Participation in leanness sports, but not training volume is associated with menstrual dysfunction: a national survey of 1276 elite athletes and controls. Br J Sports Med, 39 (2005), pp. 141-147

[8] J. Sundgot Borgen, M.K. Torstveit. Aspects of disordered eating continuum in elite high-intensity sports. Scand J Med Sci Sports, 20 (2) (2010), pp. 112-121

[9] L.K. Hilton. Loucks. Low energy availability, not exercice stress, suppresses the diurnal rythm of leptin in healthy young women. Am J Physiol, 278 (2000), pp. 43-49

[10] M.J. De Souza, D. Lee, J. VanHeest et al. Severity of energy-related menstrual disturbances increases in proportion to indices of energy conservation in exercising women. Fertil Steril, 88 (4) (2007), pp. 971-975

[11] D. Gomez-Merino, M. Chennaoui, C.Y. Guezennec. Leptine et exercice physique. Sci Sports, 19 (2004), pp. 8-18

[12] M.J. De Souza, H. Leidy, E. O’Donnel, LasleyB, I. Williams. Fasting ghrelin levels in physically active women: relationship with menstrual disturbances and metabolic hormones. J Clin Endocrinol Metab, 89 (7) (2004), pp. 3536-3542

[13] J.L. Scheid, M.J. De Souza. Menstrual irregularities and energy deficiency in physically active women: the role of ghrelin PYY and adipocytokines. Med Sport Sci, 55 (2010), pp. 82-102

[14] M. Russel, J. Strak, S. Nayak et al. Peptide YY in adolescent athletes with amenorrhea, eumenorrheic athletes and non-athletic control. Bone, 45 (2009), pp. 104-109

[15] B. Meczekalski, A. Podfigurna Stopa, A.R. Genazzani. Why kisspeptin is such important for reproduction? Gynecol Endocrinol, 27 (1) (2011), pp. 8-13

[16] K. Ackerman, K. Patel, G. Guereca. Cortisol secretory parameters in young exercisers in relation to LH secretion and bone parameters. Clin Endocrinol, 78 (1) (2013), pp. 114-119 
[17] C. Witkop, M. Warren. Understanding the spectrum of the female athlete triad. Obstet Gynecol, 116 (6) (2010), pp. 1444-1448

[18] M. Warren, A. Chua. Exercice - induced amenorrhea and bone health in the adolescent athlete. Ann N Y Acad Sci, 1135 (2008), pp. 244-252

[19] R. Ihle, A.B. Loucks. Dose reponse relationships between energy availability and bone turnover in young exercising women. J Bone Miner Res, 19 (8) (2004), pp. 1231-1240

[20] M. Russel, M. Misra. Influence of ghreline and adipocytokines on bone mineral density in adolescent female athletes with amenorrhea and eumenorrhea athletes. Med Sports Sci, 55 (2010), pp. $103-113$

[21] G. Ducher, A. Turner, S. Kukuljan et al. Obstacles in the optimization of bone health outcomes in the female athlete triad. Sports Med, 41 (7) (2011), pp. 587-607

[22] H. Lambrinoudaki, Dimitra Papadimitriou. Pathophysiology of bone loss in the female athlete. Ann N Y Acad Sci, 1205 (2010), pp. 45-50

[23] S.L. Gudmundsdottir, W.D. Flanders, Augestad L.B. Physical activity and fertility in women: the North Trondelag Health Study. Hum Reprod, 24 (12) (2009), pp. 3196-3204

[24] E. O'Donnel, M.J. De Souza. The cardiovascular effects of the chronic hypoestrogenism of the amenorrheic athletes. Sports Med, 39 (4) (2004), pp. 601-627

[25] E. Lanser, K. Zach, A. Hoch. The female athlete triad and endothelial dysfunction. Phys Med Rehabil, 3 (2011), pp. 458-465

[26] A. Rickenlund, M.J. Eriksson, K. Schenk Gustafsson, A.L. Hirschberg. Oral contraceptives improve endothelial function in amenorrheic athlete. J Clin Endocrinol Metab, 90 (2005), pp. 3162 3167 